\title{
Comparison of three assays currently used for diagnosis of legionellosis
}

Maria Chiucini, Anna Fabio, Marisa Meacci, Barbara Meccugni, Valentina Gilioli, Valeria Petocchi, Giuliana Fabio, Anna Maria Teresa Sabbatini, Sara Tagliazucchi, Sandra Mattioli, Fabio Rumpianesi

Struttura Complessa di Microbiologia e Virologia, Azienda Ospedaliero-Universitaria, Modena

Key words: Legionella pneumophila, cultures, urinary antigen, antibody research

Confronto dei test diagnostici correntemente utilizzati per la diagnosi di legionellosi

\section{SUMMARY}

The laboratory diagnosis of Legionnaires' disease is extremely complex due to the difficulty of isolating and identifying the etiological agent in a short time and also for the frequent late appearance of antibodies. For this reason it should use not only the cultures but also other diagnostic tests such as urinary antigen and antibody research.

Our study evaluated the performance of these methods. We calculated the positive predictive value (PPV) and negative predictive value (NPV), sensitivity and specificity of each test.

\section{INTRODUZIONE}

La diagnosi di laboratorio di legionellosi risulta estremamente complessa per la difficoltà di isolare e identificare l'agente eziologico in tempi brevi. ma anche per la sovente comparsa tardiva degli anticorpi (1). Per tale ragione occorre utilizzare, oltre all'isolamento colturale, altri test diagnostici come la ricerca dell'antigene urinario e la ricerca anticorpale (4). Il nostro studio è rivolto alla valutazione delle prestazioni delle suddette metodiche. A tale scopo è stato calcolato il Valore Predittivo Positivo (VPP) ed il Valore Predittivo Negativo (VPN), la sensibilità e specificità di ciascun test.

\section{MATERIALI E METODI}

Sono stati analizzati 3540 campioni biologici pervenuti presso la Struttura Complessa di Microbiologia e Virologia dell'Azienda Ospedaliero-Universitaria di Modena dal 2003 al 2009. I campioni suddetti provengono da 2547 pazienti ricoverati per sospetta infezione da L. pneumophila (Tabella $1)$.

\section{Isolamento colturale}

È stato condotto mediante l'impego di terreni di coltura specifici selettivi arricchiti: BCYE (Buffered Charcoal Yeast Extract) e MWY (Wadowsky - Yee Medium) della ditta Oxoid (Basingstoke, Hampshire, UK). Le eventuali colonie cresciute su BCYE e su MWY sono state sottoposte a sierotipizzazione mediante test di agglutinazione al lattice (Oxoid), che identifica L. pneumophila sierogruppo (sg)1, 214 e $L$. longbeachae 1-2, L. bozemanii 1-2, L. dumoffii, $L$. gormanii, L. jordanis, L. micdadei, L. anisa. In presenza di un'agglutinazione dei sg da 2-14, si è proceduto ad applicare un'ulteriore metodica per identificare lo specifico sierogruppo di L. pneumophila (Pro-Lab Diagnostics, Richmond Hill, Canada).

Antigene solubile nelle urine

L'antigene solubile urinario è stato ricercato mediante due metodiche immunocromatografiche ed una immunoenzimatica. Le metodiche immunocromatografiche sono BinaxNOW (Inverness Medical, Molenstraat, Netherlands) che permette il rilevamento qualitativo dell'antigene solubile di L. pneumophila sg1 e ?/pect legionella (Oxoid, Basingstoke, UK) che permette il rilevamento indifferenziato di L. pneumophila sg 1/sg 6. La metodica immunoenzimatica: ELISA (BIOTEST, Dreieich, Germany) permette di rilevare L. pneumophila di sg1 e sg 2-4, 6 e 10.

Ricerca di anticorpi nel siero

Il dosaggio degli anticorpi anti-Legionella di classe IgG ed IgM, eseguito mediante test ELISA Legionella pneumophila sg 1-7 (Virino/SerionGmbH, Würzburg, Germany), è stato condotto su coppie di sieri, come indicato nelle linee guida regionali (2), allo scopo di evidenziare un'eventuale sieroconversione.

\section{RISULTATI}

Come mostrato in Tabella 2, gli esiti ottenuti hanno evidenziato una negatività del $95.5 \%(3381 / 3540)$ ed una positività del 4.1\% (144/3540) per tutte le indagini eseguite. Sono stati riscontrati 15 esiti borderline di cui 14 relativi alla valutazione dell'antigenuria e uno relativo al dosaggio di IgG. L'isolamento colturale, eseguito su 458 campioni, ha evidenziato assenza di crescita di L. pneumophila nel $96.3 \%$ dei casi (441/458) e crescita nel 3.7\% (17/458); la sierotipizzazione ha evidenziato stipiti di sg 1 .

La rilevazione dell'antigene solubile urinario ha evidenziato esiti positivi pari al $3.4 \%(78 / 2314)$ e esiti negativi pari al $96.0 \%(2222 / 2314)$.

I risultati sierologici di IgG ed IgM hanno rilevato una positività per IgM nel 4.9\% (19/384) indice di infezione primaria o di permanenza delle immunoglobuline. Le IgG sono risultate positive nel $7.8 \%$ dei pazienti $(30 / 384)$ indicando una risposta specifica secondaria alle $\operatorname{IgM}$.

L'analisi degli risultati ottenuti dalle metodiche diagnostiche impiegate e dalle condizioni cliniche dei pazienti, riportate nella cartella di dimissione, permettono di quantificare il valore di ciascun test (Tabelle 3-5). A tale scopo è stato calcolato il Valore Predittivo Positivo (VPP), per valutare quanti soggetti siano stati correttamente diagnosticati come malati ed il Valore Predittivo Negativo (VPN) per valutare quanti soggetti siano stati diagnosticati correttamente come non affetti da legionellosi. Sapendo che la prevalenza della malattia è bassa, e quindi il VPP dei test non sarà mai vicino al $100 \%$, è stata appositamente valutata una popolazione a rischio di legionellosi.

Come mostrato in Tabella 3, l'isolamento colturale dimostra massima sensibilità e specificità e un VPP e un VPN corrispondenti al $100 \%$.

La Tabella 4 evidenzia che la sensibilità del test per la rilevazione dell'antigene urinario si è collocata su valori intermedi rispetto a quelli riferiti dalle specifiche del kit (75 - 99\%), mentre la specificità si è collocata nel range riferito (99100\%). È risultato ottimale il VPN superiore al VPP.

Nella Tabella 5 si riportano i dati relativi al dosaggio anticorpale; tale test ha dimostrato una ridotta sensibilità rispetto a quanto dichiarato dalla ditta produttrice che riporta valori variabili dal $70-80 \%$. La specificità è risultata elevata e superiore a quella dichiarata. Il VPP è risultato inferiore al VPN $(96.9 \%)$.

\section{Corresponding author: Maria Chiucini}

Struttura Complessa di Microbiologia e Virologia,Azienda Integrata Ospedaliero-Universitaria, Policlinico,

Via del Pozzo 7I, 4II 00 Modena - Tel.: 0594223752 - Fax: 0594223625

E-mail: chiucini@yahoo.it 
Tabella I. Numero di esami suddivisi per tipologia di indagine

\begin{tabular}{lllll}
\hline Periodo 2003-2009 Isolamento colturale materiali respiratori & Antigene urinario Sierologia IgG/IgM & Totale
\end{tabular}

\begin{tabular}{lllll}
$\mathbf{N}^{\circ}$ esami & 458 & $23 / 4$ & $384 / 384$ & 3540 \\
\hline
\end{tabular}

Tabella 2. Risultati ottenuti suddivisi per tipologia di esame

\begin{tabular}{|c|c|c|c|c|}
\hline Periodo 2003 - 2009 & Esito positivo & Esito negativo & Esito borderline & Totale esami \\
\hline $\begin{array}{l}\text { Isolamento.colturale } \\
\text { materiali respiratori }\end{array}$ & 17 (3.7\%) & $44 I(96.3 \%)$ & l & $458(13 \%)$ \\
\hline Ag urinario & $78(3.4 \%)$ & $2222(96.0 \%)$ & $14(0.6 \%)$ & $23 \mid 4(65.4 \%)$ \\
\hline Anti-Legionella IgG & $30(7.8 \%)$ & $353 \quad(91.9 \%)$ & I $(0.3 \%)$ & $384(10.8 \%)$ \\
\hline Anti-Legionella IgM & $19(4.9 \%)$ & $365 \quad(95.1 \%)$ & 1 & $384(10.8 \%)$ \\
\hline Totale & I44 (4. I\%) & $338 I(95.5 \%)$ & I $5(0.4 \%)$ & 3540 \\
\hline
\end{tabular}

Tabella 3. Validità dell'isolamento colturale

\begin{tabular}{|c|c|c|c|c|c|c|}
\hline \multirow[b]{2}{*}{ Positivi } & \multicolumn{2}{|c|}{ Malati } & \multicolumn{2}{|c|}{ Sani } & \multicolumn{2}{|c|}{ Totale } \\
\hline & $\mathrm{a}(\mathrm{VP})$ & 17 & $\mathrm{~b}(\mathrm{FP})$ & 0 & $a+b$ & 17 \\
\hline Negativi & c (FN) & 0 & $\mathrm{~d}(\mathrm{VN})$ & 441 & $c+d$ & 441 \\
\hline Totale & $a+c$ & 17 & $b+d$ & 441 & $a+b+c+d$ & 458 \\
\hline \multicolumn{3}{|c|}{ Sensibilità: $a /(a+c)$} & \multicolumn{2}{|c|}{$17 / 17=100 \%$} & & \\
\hline \multicolumn{2}{|c|}{ Specificità: $d /(b+d)$} & & \multicolumn{2}{|c|}{$44|/ 44|=100 \%$} & & \\
\hline \multicolumn{2}{|c|}{ VPP: $a /(a+b)$} & & \multicolumn{2}{|c|}{$17 / 17=100 \%$} & & \\
\hline \multicolumn{2}{|c|}{ VPN: $d /(c+d)$} & & \multicolumn{2}{|c|}{$44 I / 44 I=100 \%$} & & \\
\hline
\end{tabular}

Tabella 4. Validità della rilevazione dell'antigene urinario

\begin{tabular}{llllllr}
\hline \multicolumn{3}{c}{ Malati } & \multicolumn{2}{c}{ Sani } & \multicolumn{2}{c}{ Totale } \\
\hline Positivi & $\mathrm{a}(\mathrm{VP})$ & 69 & $\mathrm{~b}(\mathrm{FP})$ & 9 & $\mathrm{a}+\mathrm{b}$ & 78 \\
\hline Negativi & $\mathrm{c}(\mathrm{FN})$ & $\mathrm{I} 5$ & $\mathrm{~d}(\mathrm{VN}) 222 \mathrm{l}$ & $\mathrm{c}+\mathrm{d}$ & 2236 \\
\hline Totale & $\mathrm{a}+\mathrm{c}$ & 84 & $\mathrm{~b}+\mathrm{d}$ & 2230 & $\mathrm{a}+\mathrm{b}+\mathrm{c}+\mathrm{d}$ & $23 \mathrm{I4}$ \\
\hline Sensibilità: $\mathrm{a} /(\mathrm{a}+\mathrm{c})$ & & $69 / 84=82.1 \%$ & & \\
Specificità: $\mathrm{d} /(\mathrm{b}+\mathrm{d})$ & & $2221 / 2230=99.6 \%$ & & \\
VPP: $\mathrm{a} /(\mathrm{a}+\mathrm{b})$ & $69 / 78=88.5 \%$ & & \\
VPN: $\mathrm{d} /(\mathrm{c}+\mathrm{d})$ & & $2221 / 2236=99.3 \%$ & &
\end{tabular}

Tabella 5. Validità del dosaggio anticorpale (IgM e lgG)

\begin{tabular}{|c|c|c|c|c|c|c|}
\hline & \multicolumn{2}{|c|}{ Malati } & \multicolumn{2}{|c|}{ Sani } & \multicolumn{2}{|c|}{ Totale } \\
\hline Positivi & a (VP) & 37 & b (FP) & 12 & $a+b$ & 49 \\
\hline Negativi & c (FN) & 22 & $d(V N)$ & 697 & $c+d$ & 719 \\
\hline Totale & $a+c$ & 59 & $b+d$ & 709 & $a+b+c+d$ & 768 \\
\hline \multicolumn{2}{|c|}{ Sensibilità: $a /(a+c)$} & \multicolumn{3}{|c|}{$37 / 59=62.7 \%$} & & \\
\hline \multicolumn{2}{|c|}{ Specificità: $d /(b+d)$} & \multicolumn{3}{|c|}{$697 / 709=98.3 \%$} & & \\
\hline \multicolumn{2}{|c|}{ VPP: $a /(a+b)$} & \multicolumn{3}{|c|}{$37 / 49=75.5 \%$} & & \\
\hline \multicolumn{2}{|c|}{ VPN: $d /(c+d)$} & \multicolumn{3}{|c|}{$697 / 719=96.9 \%$} & & \\
\hline
\end{tabular}

\section{DISCUSSIONE}

Il test per la ricerca di antigene urinario ha presentato ottima specificità e buona sensibilità con elevato VPN e quindi ha dimostrato di individuare correttamente il maggior numero di soggetti non affetti da legionellosi.

Gli esiti relativi al dosaggio anticorpale hanno mostrato bassa sensibilità; il VPN è risultato ottimale mentre il VPP è risultato inferiore probabilmente, come indicato dalla ditta produttrice, a causa di cross-reattività con altri agenti patogeni.

I risultati ottenuti dall'isolamento colturale hanno dimostrato la più elevata sensibilità e specificità ed ottimo VPP e VPN; pertanto l'isolamento colturale si riconferma il gold standard diagnostico ma, per la non facile reperibilità dei campioni e i tempi relativamente lunghi di crescita delle colonie, deve essere affiancato dalle altre metodiche descritte ai fini di una più rapida e completa definizione etiologica della malattia (3).

\section{BIBLIOGRAFIA}

1. Bartram J, Chartier Y, Lee J, Pond K, Surman-Lee S. Legionella and the prevention of legionellosis. World Health Organizations 2007.

2. Bollettino Ufficiale RER. Deliberazione della Giunta Regionale 21 luglio 2008 , n.1115. Approvazione linee guida regionali per la sorveglianza e il controllo della legionellosi.

3. Fields BS, Benson RF, Besser RE. Legionella and Legionnaire's disease: 25 years of investigation. Clin Microbio Rev 2002; 15 (3): 506-26.

4. Stout JE, Rihs JD, Yu VL. Legionella. In: Manual of clinical microbiology, Murray PR, et al. Eds., 8th Edition., ASM Press, Washington DC 2003; 809-82. 